\title{
Clinical and microbiological characteristics of bloodstream infections caused by Enterococcus spp. within internal medicine wards: a two-year single-centre experience
}

\author{
Tommaso Lupia $^{1}$ (1) - Gianmario Roberto ${ }^{2} \cdot$ Luca Scaglione $^{2} \cdot$ Nour Shbaklo $^{3} \cdot$ llaria De benedetto ${ }^{3} \cdot$ Silvia Scabini $^{3}$. \\ Simone Mornese Pinna ${ }^{3} \cdot$ Antonio Curtoni $^{4} \cdot$ Rossana Cavallo ${ }^{4}$. Francesco Giuseppe De Rosa ${ }^{1,3} \cdot$ Silvia Corcione ${ }^{1,5}$
}

Received: 21 July 2021 / Accepted: 4 January 2022 / Published online: 29 January 2022

(c) The Author(s), under exclusive licence to Società Italiana di Medicina Interna (SIMI) 2022

\begin{abstract}
Enterococcal bloodstream infections (E-BSI) constitute the second cause of Gram-positive bacterial BSI in Europe with a high rate of in-hospital mortality. Furthermore, E-BSI treatment is still challenging because of intrinsic and acquired antibiotic resistances. We conducted a retrospective, 2-year, observational, single-centre study to evaluate clinical outcome and risk factors for E-BSI mortality in internal medicine wards. 201 patients with E-BSI were included in the analysis. Infection rate was 2.4/1000 days of hospital admission. Most E-BSI were hospital acquired (78.1\%). The median age was 68 years. Charlson Comorbidity Index, adjusted for age, was 5 (range 4-6). Patients with E-BSI frequently had at least one invasive device, predominantly a central venous (73\%) or a bladder catheter $(61.7 \%)$. Enterococcus faecium accounted for $47.94 \%$ of E-BSI (resistance rate to ampicillin or vancomycin was 22.2 and 23.3\%, respectively) and Enterococcus faecalis for 52.08\% (resistance rate to ampicillin or vancomycin was 3.1 and 2.2\%, respectively). Among all E-BSI, 25\% of patients received appropriate therapy. In total, 59\% of E-BSI underwent echocardiography. At the multivariate analysis, resistance to vancomycin (OR 2.09, $p=0.025$ ), sepsis (OR 2.57, $p=0.003$ ) and septic shock (OR 3.82, $p=0.004$ ) was a predictor of mortality. No difference in 28-day survival was observed between appropriate or inappropriate treatment, except for endocarditis. However, E-BSI sources in clinical practices are not always properly investigated, including the rule-out of intracardiac vegetations. We did not demonstrate a difference in mortality for inappropriate therapy in the absence of endocarditis in comorbid patients with a long history of medicalization.
\end{abstract}

Keywords Enterococci $\cdot$ Bloodstream infections $\cdot$ faecalis $\cdot$ faecium $\cdot$ Internal medicine wards

Tommaso Lupia

tommaso.lupia89@gmail.com

1 Unit of Infectious Diseases, Cardinal Massaia Hospital, Asti, Italy

2 Unit of Internal Medicine, Department of Medicine, Città della Salute e della Scienza, Turin, Italy

3 Department of Medical Sciences, Infectious Diseases, University of Turin, Turin, Italy

4 Microbiology and Virology Unit, AOU Città della Salute e della Scienza di Torino, Turin, Italy

5 Tufts University School of Medicine, Boston, USA

\section{Introduction}

Enterococcus faecalis and Enterococcus faecium have become increasingly common causes of healthcare-associated infections (HAIs) in the United States (US) and Europe [1-3]. Six-year surveillance on Gram-positive organisms causing bacteraemia in US and European hospitals reported that $10.6 \%$ and $9.4 \%$ of bloodstream infections (BSI) were due to E. faecalis and E. faecium, which constitute, respectively, the second and the third pathogen in terms of frequency after Staphylococcus aureus [4]

The incidence of enterococci as the causative agent of BSI (E-BSI) continues to be also elevated during the COVID-19 pandemic, probably because of a more extensive empirical use of corticosteroids and cephalosporins which have variable in vivo activity against Enterococcus spp. [5]. In COVID-19 subjects, E. faecalis and E. faecium represented 
$18 \%$ and $9 \%$ of all BSI, according to a recent retrospective study by Giacobbe et al. [5].

Moreover, it is well established that E-BSI retained high in-hospital mortality estimated between 30 to $50 \%$ [6-10] and it has been identified as an independent predictor of increased 30-day mortality in elderly patients [11].

Furthermore, enterococci also play an increasingly important role in infective endocarditis, being the third leading causative agent in high-income countries and more frequently occurring in aged patients with high complexity of comorbidities [12].

Antibiotic combination is usually needed to achieve bactericidal activity in enterococcal-related endocarditis, although the possibility of combining antibiotics has become more challenging due to the increasing rate of acquired antimicrobial resistance among enterococci [13, 14]. Antibiotics' timing and selection represent another critical issue, since two meta-analyses showed that inappropriate and delayed therapy in clinical practice is associated with more unfavourable outcomes and higher mortality [13, 14].

This retrospective study aimed to evaluate the clinical outcome and risk factors for mortality of patients with E-BSI admitted to internal medicine wards (IMW) in a large teaching hospital in northwest Italy over 2 years.

\section{Methods}

This retrospective study was conducted in IMWs, in a 1200bed academic hospital with a primary and secondary referral (City of Health and Sciences, Molinette, Turin, Italy).

All adults with positive blood culture for E. faecalis or $E$. faecium between 1st January 2015 and 31st December 2016 were enrolled in the study, and the demographic, clinical and microbiological data were collected.

BSI were defined as community acquired (CA-BSI), hospital acquired (HA-BSI) or healthcare associated (HCABSI), according to the time and setting of the onset of infection. HA-BSI was defined as a first positive blood culture $\geq 48 \mathrm{~h}$ after hospital admission or within $48 \mathrm{~h}$ of discharge from the hospital. HCA-BSI was defined as a first positive blood culture obtained $<48 \mathrm{~h}$ of admission in patients with recent and/or frequent exposure to healthcare settings and procedures. Community-acquired infections are those with a first positive culture obtained $<48 \mathrm{~h}$ of admission that do not fulfil criteria for the healthcare-associated classification.

The Sequential Organ Failure Assessment (SOFA) score and quick-SOFA (q-SOFA) were used to assess the severity of the disease [15], and the Charlson Comorbidity Index [16], a weighted index of 17 coexisting medical conditions, was used as the measure of the risk of mortality of each comorbidity in this population.
According to Sepsis-2 guideline [17], sepsis was defined as the presence of at least two systemic inflammatory response syndrome (SIRS) criteria. Severe sepsis was defined accordingly as Sepsis-2, and sepsis was defined according to Sepsis-3 guideline [15].

Antibiotic therapy was defined as appropriate if all the following criteria were present: the administration of an active antibiotic against Enterococcus spp., the administration of an active combination therapy (at least two drugs simultaneously, except daptomycin) in infective endocarditis and a duration of at least 15 days of an active antibiotic treatment [18-21]. Antimicrobials considered active in monotherapy or in combination therapy against Enterococcus spp. were penicillin G, ampicillin, ampicillin/sulbactam, amoxicillin, amoxicillin/clavulanate, piperacillin/tazobactam, imipenem, vancomycin, teicoplanin, daptomycin, ceftriaxone, gentamicin, tigecycline and linezolid.

In case of death or discharge within 15 days from the beginning of antibiotic therapy, the therapy was considered appropriate if criteria 1 and 2 were satisfied. Moreover, the 21-day mortality was investigated.

The Vitek MS MALDI-TOF mass spectrometry (bioM erieux, Craponne, France) was routinely used for identifying Enterococcus spp. BSI, whereas the Vitek 2 automated system (bioMerieux, Craponne, France) was routinely used for susceptibility testing. The results of the susceptibility tests were interpreted according to the criteria of the European Committee on Antimicrobial Susceptibility Testing (EUCAST) (breakpoint tables for interpretation of minimum inhibitory concentrations $[\mathrm{MIC}]$ and zone diameters, version 10.0, 2020; http://www.eucast.org). For daptomycin, isolates were considered susceptible in the case of MIC $4 \mathrm{mg} / \mathrm{L}$. Specifically, daptomycin was used at high dose, in combination therapy, in Enterococcus spp. strains reporting MIC $<4 \mathrm{mg} / \mathrm{L}$.

The need for informed consent was waived due to the study's retrospective nature, which the medical direction of the hospital approved (PROT.N.0115709). Data were collected according to the Italian laws on privacy.

At the time of blood cultures, demographic and clinical characteristics were summarized using absolute and percentage frequencies (qualitative variables) or using the mean and standard deviation or percentile (quantitative variables). Categorical variables were tested in the Chi-square test. Numeric variables were tested by Student's $t$ test or Wilcoxon test. Overall survival was defined as the time between the first blood sample culture and hospital death. The equivalence of survival curves was tested through the log-rank test. A Cox proportional-hazards model was used for multivariate analysis. Statistical analysis was made using STATA (StataCorp. 2017. Stata Statistical Software: Release 15. College Station, TX: StataCorp LP). 


\section{Results}

Two hundred and eighteen episodes of E-BSI were included in the study. Due to incomplete medical records, the analysis was performed on 201 patients. The infection rate was 2.4/1000 days of hospital admission. Most E-BSIs were nosocomial related (78.1\% HA-BSI, $12.4 \%$ HCA and $8.9 \% \mathrm{CA})$.

As shown in Table 1, E-BSI occurred mainly in female $(61.7 \%)$, with a median age of 68 .

$98.5 \%$ of all patients had comorbidities, and the median Charlson Comorbidity Index, adjusted for age, was five (range between four and six). Cancer was the most common comorbidity $(N=67,33.3 \%)$, followed by diabetes $(N=52,25.9 \%)$ and chronic obstructive pulmonary disease (COPD) $(N=51 ; 25.4 \%)$. Approximately 117 patients (58.5\%) were hospitalized during the previous 3 months $(67.8 \%$ in a medical ward, $12.9 \%$ in a surgical ward and $21.4 \%$ in an intensive care unit).

Patients with E-BSI frequently had invasive devices, mainly a central venous catheter (CVC) (73\%) or a urinary catheter $(61.7 \%)$ (Table 1$)$.

$21 \%$ of patients were on immunosuppressive therapy other than chronic corticosteroid treatment $(12.4 \%)$ or had undergone solid organ or hematopoietic stem cell transplant ( $9.4 \%$ and $1.5 \%$, respectively).

Among all E-BSI cases, in $47.9 \%$, E. faecium was responsible, whereas E. faecalis was isolated in $52.1 \%$. E. faecalis was resistant to ampicillin $(N=7 ; 3.1 \%)$ or vancomycin-resistant enterococci (VRE) $N=5 ; 2.2 \%$. On the other hand, E. faecium were ampicillin resistant in $22.2 \%$ and VRE strains were $23.3 \%(N=39)$.

Regarding the source of infection, abdominal infections were the most frequent $(21.2 \%)$, followed by pulmonary infections $(18.0 \%)$ and urinary tract infections $(16.0 \%)$, according to clinical presentation and radiological features. Infective endocarditis was diagnosed in $5.5 \%$, but overall only $58.6 \%$ of patients did not undergo echocardiography.

Following Sepsis-2 criteria, sepsis occurred in 41.7\% of participants and septic shock in $8.0 \%$. Data on severe sepsis have not been analysed for lack of complete data.

According to Sepsis-3 criteria, $15.9 \%$ of participants developed sepsis, and 5.4\% developed septic shock.

Fifty-two patients (25.8\%) received appropriate therapy. The most frequent antibiotic used was vancomycin (17.8\%), followed by piperacillin/tazobactam (15.9\%) alone or in combination (Table 2).

\section{Risk factors for mortality}

According to the univariate analysis (Table 3), mortality was associated with resistance to vancomycin $(p=0.042$, 95\% CI $[1.02,3.31])$, sepsis $(p=0.003,95 \%$ CI $[1.36$, $4.58])$ and septic shock ( $p=0.007,95 \%$ CI $[1.39,8.05])$. SIRS presentation, instead, was linked to lower mortality $(p=0.047,95 \%$ CI $[1.39,8.05])$. The multivariate analysis (Table 4) confirmed resistance to vancomycin (OR 2.09, $95 \%$ CI [1.10, 3.99], $p=0.025$ ), sepsis (OR 2.57, 95\% CI $[1.37,4.82], p=0.003)$ and septic shock (OR 3.82, 95\% CI $[1.53,9.55], p=0.004)$ as risk factors for death and sepsis, according to Sepsis-2 criteria as a protective factor (OR $0.50,95 \%$ CI [0.26, 0.99], $p=0.047$ ).

\section{Survival}

No difference was found between the group that received appropriate therapy and the group that did not receive appropriate therapy, except for endocarditis, which was more frequent in the first group $(p=0.003)$.

During hospitalization, 53 people (26\%) died. Their median survival time was 65 days after the first blood draw. The other $75.4 \%$ lived at least 21 days, as shown by the Kaplan-Meier curve. After 28 days of follow-up, the survivorship curve showed no difference between the patients who received appropriate treatment and the patients who did not (Fig. 1).

\section{Discussion}

Enterococci are Gram-positive pathogens frequently involved in BSI related to high in-hospital mortality, especially in high comorbid, frail patients $[6,7]$. There is a high burden of widespread, difficult-to-treat multi-drug-resistant E. faecium, and E. faecalis is increasingly resistant to firstline antibiotic choices [7, 8]. While evidence suggests that combination therapy can decrease mortality in patients with enterococcal infective endocarditis, there is no strong evidence on the best therapy for patients with an E-BSI without endocarditis $[13,14]$.

We retrospectively reported epidemiological, microbiological and clinical features of the population with E-BSI in a single-centre, 2-year observational study. In our study, almost $80 \%$ of E-BSIs were hospital acquired, whereas, in similar studies, the incidence was about $40 \%$ [7, 22]. Nonetheless, the healthcare-associated incidence of E-BSI was much lower in our study than that in recent literature (about $10 \%$ vs $45 \%$ ) [22]. Moreover, as previously described [7, 8, 22], we observed that BSI frequently occur in the elderly with several comorbidities. Among comorbidities, solid tumour, cardiovascular diseases and diabetes were the three 
Table 1 Descriptive analysis of demographic, clinical and microbiological data sorted according to the appropriateness of treatment in bloodstream infections due to Enterococcus spp.

\begin{tabular}{|c|c|c|c|c|c|c|c|}
\hline & \multicolumn{2}{|c|}{$\begin{array}{l}\text { Appropriate treat- } \\
\text { ment }(N=149) \\
\end{array}$} & \multicolumn{2}{|c|}{$\begin{array}{l}\text { Inappropriate treat- } \\
\text { ment }(N=52)\end{array}$} & \multicolumn{2}{|c|}{ Total $(N=201)$} & \multirow[t]{2}{*}{$p$-value } \\
\hline & No. & $\%$ & No. & $\%$ & No. & $\%$ & \\
\hline Sex & & & & & & & 0.333 \\
\hline Female & 89 & 59.73 & 35 & 67.31 & 124 & 61.69 & \\
\hline Male & 60 & 40.27 & 17 & 32.69 & 77 & 38.31 & \\
\hline Age_-average (SD) & 69.95 & 12.65 & 68.65 & 13.90 & 68.02 & 12.96 & $0.537^{\mathrm{a}}$ \\
\hline Comorbidities (at least 1) & 171 & 99.30 & 57 & 96.61 & 198 & 98.51 & 0.153 \\
\hline Diabetes & 37 & 24.83 & 15 & 28.85 & 52 & 25.87 & 0.569 \\
\hline Liver disease & 21 & 14.09 & 5 & 9.62 & 26 & 12.94 & 0.407 \\
\hline Cancer & 45 & 30.2 & 22 & 42.31 & 67 & 33.33 & 0.111 \\
\hline AIDS & 0 & 0 & 0 & 0 & 0 & 0 & \\
\hline Kidney disease & 26 & 17.45 & 13 & 25 & 39 & 19.4 & 0.236 \\
\hline Hearth failure & 26 & 17.45 & 11 & 21.15 & 37 & 18.41 & 0.553 \\
\hline Myocardial infraction & 31 & 20.81 & 11 & 21.15 & 42 & 20.9 & 0.958 \\
\hline COPD & 38 & 25.5 & 13 & 25 & 51 & 25.37 & 0.943 \\
\hline Peripheral vascular disease & 22 & 14.77 & 7 & 13.46 & 29 & 14.43 & 0.818 \\
\hline Stroke & 22 & 14.77 & 5 & 9.62 & 27 & 13.43 & 0.348 \\
\hline Hemiplegia & 10 & 6.71 & 3 & 5.77 & 13 & 6.47 & 0.812 \\
\hline Dementia & 11 & 7.38 & 7 & 13.46 & 18 & 8.96 & 0.186 \\
\hline Rheumatologic disease & 4 & 2.68 & 0 & 0 & 4 & 1.99 & 0.233 \\
\hline Peptic ulcer & 6 & 4.03 & 0 & 0 & 6 & 2.99 & 0.142 \\
\hline Charlson Index —-median (interquartile range) & 5 & $(4-5)$ & 6 & $(5-6)$ & 5 & $(4-6)$ & $0.111^{\mathrm{b}}$ \\
\hline \multicolumn{8}{|l|}{ Risk factors for infection } \\
\hline Hospitalization (last 3 months) ${ }^{c}$ & 83 & 56.08 & 34 & 65.38 & 117 & 58.5 & 0.241 \\
\hline Antibiotic therapy (last 6 months) ${ }^{d}$ & 74 & 50.34 & 26 & 50.98 & 100 & 50.51 & 0.937 \\
\hline Surgical procedure (last 6 months) & 57 & 38.26 & 17 & 32.69 & 74 & 36.82 & 0.474 \\
\hline Abdominal surgical drain (last 6 months) ${ }^{e}$ & 25 & 17.01 & 9 & 17.31 & 34 & 17.09 & 0.960 \\
\hline Abdominal trauma & 2 & 1.34 & 0 & 0 & 2 & 1 & 0.401 \\
\hline Use of steroids & 19 & 12.75 & 6 & 11.54 & 25 & 12.44 & 0.819 \\
\hline Solid organ transplant & 16 & 10.74 & 3 & 5.77 & 19 & 9.45 & 0.292 \\
\hline Stem cell transplant & 2 & 1.34 & 1 & 1.92 & 3 & 1.49 & 0.766 \\
\hline Ileocolostomy & 6 & 4.03 & 3 & 5.77 & 9 & 4.48 & 0.601 \\
\hline Percutaneous endoscopic gastrostomy ${ }^{f}$ & 8 & 5.41 & 3 & 5.77 & 11 & 5.50 & 0.921 \\
\hline Parenteral nutrition & 43 & 28.86 & 14 & 26.92 & 57 & 28.36 & 0.790 \\
\hline Enteral nutrition & 31 & 20.81 & 11 & 21.15 & 42 & 20.90 & 0.958 \\
\hline Immunosuppressive therapy ${ }^{\mathrm{g}}$ & 31 & 20.95 & 11 & 21.15 & 42 & 21.00 & 0.975 \\
\hline Mechanical ventilation & & & & & & & 0.265 \\
\hline No & 104 & 71.23 & 36 & 70.59 & 140 & 71.07 & \\
\hline Endotracheal & 34 & 23.29 & 9 & 17.65 & 43 & 21.83 & \\
\hline Non-invasive & 8 & 5.48 & 6 & 11.76 & 14 & 7.11 & \\
\hline Intravenous catheter & 94 & 63.09 & 30 & 57.69 & 124 & 61.69 & 0.491 \\
\hline Central line catheter ${ }^{\mathrm{h}}$ & 109 & 73.65 & 37 & 71.15 & 146 & 73 & 0.727 \\
\hline \multicolumn{8}{|l|}{ Source of infection } \\
\hline Urinary tract infection ${ }^{\mathrm{i}}$ & 28 & 18.92 & 4 & 7.69 & 32 & 16.00 & 0.057 \\
\hline Pulmonary infection ${ }^{j}$ & 27 & 18.24 & 9 & 17.31 & 36 & 18.00 & 0.880 \\
\hline Bile duct infection ${ }^{\mathrm{k}}$ & 18 & 12.16 & 5 & 9.62 & 23 & 11.50 & 0.620 \\
\hline Intraabdominal infection ${ }^{1}$ & 5 & 3.38 & 4 & 7.84 & 9 & 4.52 & 0.186 \\
\hline Gastroenteritis $^{\mathrm{m}}$ & 1 & 0.68 & 2 & 3.85 & 3 & 1.50 & 0.106 \\
\hline Surgical site infection ${ }^{n}$ & 4 & 2.7 & 1 & 1.92 & 5 & 2.50 & 0.757 \\
\hline Diabetic foot ${ }^{\circ}$ & 3 & 2.03 & 0 & 0 & 3 & 1.50 & 0.301 \\
\hline Cellulitis $^{p}$ & 1 & 0.68 & 0 & 0 & 1 & 0.50 & 0.551 \\
\hline Infected decubitus ${ }^{\mathrm{q}}$ & 8 & 5.48 & 5 & 9.8 & 13 & 6.60 & 0.284 \\
\hline Echocardiography & & & & & & & 0.003 \\
\hline Not executed & 91 & 61.9 & 25 & 49.02 & 116 & 58.59 & \\
\hline Negative & 52 & 35.37 & 18 & 35.29 & 70 & 35.35 & \\
\hline Positive for endocarditis & 3 & 2.04 & 8 & 15.69 & 11 & 5.56 & \\
\hline Diagnosis of endocarditis with negative echocardiography & 1 & 0.68 & 0 & 0 & 1 & 0.51 & \\
\hline Type of acquisition & & & & & & & 0.942 \\
\hline Community acquired & 13 & 8.72 & 5 & 9.62 & 18 & 8.96 & \\
\hline Healthcare associated & 18 & 12.08 & 7 & 13.46 & 25 & 12.44 & \\
\hline
\end{tabular}


Table 1 (continued)

\begin{tabular}{|c|c|c|c|c|c|c|c|}
\hline & \multicolumn{2}{|c|}{$\begin{array}{l}\text { Appropriate treat- } \\
\text { ment }(N=149) \\
\end{array}$} & \multicolumn{2}{|c|}{$\begin{array}{l}\text { Inappropriate treat- } \\
\text { ment }(N=52)\end{array}$} & \multicolumn{2}{|c|}{ Total $(N=201)$} & \multirow[t]{2}{*}{$p$-value } \\
\hline & No. & $\%$ & No. & $\%$ & No. & $\%$ & \\
\hline Nosocomial acquired & 118 & 79.19 & 40 & 76.92 & 158 & 78.61 & \\
\hline \multicolumn{8}{|l|}{ Clinical presentation } \\
\hline Sepsis-3 criteria & & & & & & & 0.292 \\
\hline No & 116 & 77.85 & 42 & 80.77 & 158 & 78.61 & \\
\hline Shock & 9 & 6.04 & 2 & 3.85 & 11 & 5.47 & \\
\hline Sepsis & 24 & 16.11 & 8 & 15.38 & 32 & 15.92 & \\
\hline Sepsis-2 criteria & & & & & & & 0.318 \\
\hline No & 75 & 51.02 & 25 & 48.08 & 100 & 50.25 & \\
\hline Shock & 14 & 9.52 & 2 & 3.85 & 16 & 8.04 & \\
\hline Sepsis & 58 & 39.46 & 25 & 48.08 & 83 & 41.71 & \\
\hline Severe sepsis & $\mathrm{NA}$ & $\mathrm{NA}$ & NA & NA & $\mathrm{NA}$ & NA & \\
\hline \multicolumn{8}{|l|}{ Microbiological data } \\
\hline Polymicrobial & 45 & 30.2 & 14 & 26.92 & 59 & 29.35 & 0.655 \\
\hline Enterococcus faecium ${ }^{\mathrm{r}}$ & 64 & 45.07 & 29 & 55.77 & 93 & 47.94 & 0.186 \\
\hline Ampicillin resistant $^{\S}$ & 61 & 41.78 & 27 & 51.92 & 88 & 44.44 & 0.206 \\
\hline Gentamicin resistant $^{t}$ & 62 & 42.47 & 28 & 53.85 & 90 & 45.45 & 0.157 \\
\hline Vancomycin resistant ${ }^{\mathrm{u}}$ & 23 & 15.97 & 14 & 27.45 & 37 & 18.97 & 0.072 \\
\hline
\end{tabular}

${ }^{\mathrm{a}} T$ test $p$ value

${ }^{\mathrm{b}}$ Wilcoxon test $p$ value

${ }^{\mathrm{c}} N=200$

${ }^{\mathrm{d}} N=198$

${ }^{\mathrm{e}} N=199$

${ }^{\mathrm{f}} N=200$

${ }^{\mathrm{g}} N=200$

${ }^{\mathrm{h}} N=200$

${ }^{\mathrm{i}} N=200$

${ }^{\mathrm{j}} N=200$

${ }^{\mathrm{k}} N=200$

${ }^{1} N=199$

${ }^{\mathrm{m}} N=200$

${ }^{\mathrm{n}} N=200$

${ }^{\circ} N=200$

${ }^{\mathrm{p}} N=199$

${ }^{\mathrm{q}} N=197$

${ }^{\mathrm{r}} N=198$

${ }^{\mathrm{s}} N=199$

${ }^{\mathrm{t}} N=198$

${ }^{\mathrm{u}} N=195$ most frequent underlying diseases. Interestingly in this series, COPD (25.3\%) was reported at a higher rate than previous studies [7,9]. This data might be explained, according to literature, by a frequent need for hospitalization, chronic corticosteroid treatment, and airways and gut bacterial colonization in people with COPD accounting for a higher risk of E-BSI. Inghammar et al. [23] described a 2.5-fold increase in bacteraemia in the COPD population in Sweden, and among pathogens involved, E-BSI was more frequent than in the control group [HR 1.9, 95\% CI $(1.3,2.6)]$. In previous works, COPD patients, notably in the ICU, reported frequent VRE colonization and higher related BSI rates [24, 25]. Moreover, a high comorbidity burden such as the one in our population with a median Charlson Comorbidity Index (CCI) of 5 must be taken into account in prognostic considerations given that in E-BSI a Charlson Comorbidity Index (CCI) higher than four has been independently associated with 30-day mortality [26].

Iatrogenic immunosuppression is a common risk for enterococcal-related infections, with up to $33 \%$ of these patients being admitted to receiving immunosuppressive therapy other than chronic corticosteroids, and $9.4 \%$ being 
Table 2 Antibiotic molecules used in monotherapy or in combination during empirical or targeted therapy in bloodstream infections due to Enterococcus spp.

\begin{tabular}{ll}
\hline Antibiotic & $N(\%)$ \\
\hline Vancomycin & $106(17.8)$ \\
Piperacillin/tazobactam & $95(15.9)$ \\
Linezolid & $53(8.9)$ \\
Ceftriaxone & $30(5.0)$ \\
Gentamicin & $25(4.2)$ \\
Tigecycline & $25(4.2)$ \\
Daptomycin & $24(4.0)$ \\
Ampicillin & $22(3.7)$ \\
Ampicillin/sulbactam & $17(2.8)$ \\
Teicoplanin & $12(2.0)$ \\
Amoxicillin/clavulanate & $11(1.9)$ \\
Imipenem & $8(1.3)$ \\
\hline
\end{tabular}

Table 3 Univariate analysis of risk factors for 21-day mortality associated with Enterococcus spp. bacteraemia

\begin{tabular}{|c|c|c|}
\hline & HR & CI \\
\hline Appropriated therapy & 1.25 & {$[0.70,2.23] 0.452$} \\
\hline Sex & 0.98 & {$[0.56,1.73] 0.956$} \\
\hline Age & 1 & {$[0.98,1.02] 0.976$} \\
\hline Charlson Index & 1.1 & {$[0.99,1.22] 0.084$} \\
\hline Solid organ transplant & 0.9 & {$[0.36,2.26] 0.819$} \\
\hline Use of steroids & 1.53 & {$[0.77,3.04] 0.228$} \\
\hline Polymicrobial & 0.6 & {$[0.32,1.13] 0.113$} \\
\hline E. faecium vs. E. faecalis & 1.14 & {$[0.66,1.95] 0.638$} \\
\hline Ampicillin-resistant strains & 1.23 & {$[0.72,2.11] 0.454$} \\
\hline Gentamicin-resistant strains & 1.55 & {$[0.90,2.68] 0.114$} \\
\hline Vancomycin-resistant strains & 1.84 & {$[1.02,3.31] 0.042$} \\
\hline Medical ward & 1 & {$[1.00,1.00]-$} \\
\hline Surgical ward & 1.18 & {$[0.52,2.71] 0.694$} \\
\hline Intensive care unit & 1.62 & {$[0.89,2.96] 0.115$} \\
\hline Previous stay in intensive care unit & 0.98 & {$[0.39,2.46] 0.968$} \\
\hline Shock (sepsis 3) & 3.34 & {$[1.39,8.05] 0.007$} \\
\hline Sepsis (sepsis 3) & 2.29 & {$[1.36,4.58] 0.003$} \\
\hline Shock (sepsis 2) & 1.91 & {$[0.91,4.02] 0.087$} \\
\hline Severe sepsis & - & - \\
\hline Sepsis (sepsis 2) & 0.49 & {$[0.25,0.97] 0.047$} \\
\hline
\end{tabular}

admitted to receiving procedures resulting in more aggressive immune-suppression such as solid organ transplant. Besides, in COVID-19 subjects, the acute use of anti-inflammatory agents has been recently identified as an independent predictor of the development of E-BSI [4].

In our cohort, up to $54.7 \%$ of participants received enteral or parenteral nutrition during hospitalization. Enteral feeding has reported previously contrasting findings as a protective
[23] or risk factor [7] for enterococcal infection, whereas parenteral nutrition is a well-known factor associated with catheter-related BSI in patients with central venous catheters (CVC) used to administer parenteral nutrition [28-30]. It is relevant to consider that the relative majority, almost one third, of our population received parenteral nutrition. Also, almost $80 \%$ of patients had a CVC. The primary source of infections was intraabdominal, including peritonitis, gastroenteritis and biliary tract infections, as stated in several studies [7-9, 24-27].

We also described a surprisingly high proportion of infection supposed to originate from the respiratory tract. Several authors have suggested a correlation between enterococcal pneumonia and enteral feeding, based on a higher risk of oropharynx colonization and the use of broad-spectrum antibiotics [28-30]. In this study, the number of BSI with pulmonary sources (18\%) was the highest reported, to our knowledge, with more than $50 \%$ of the total population reporting an antibiotic treatment in the previous 6 months and more than $20 \%$ having been submitted previously to IMW admission to invasive mechanical ventilation.

Enterococcus spp. is commonly known as a healthcareassociated pathogen, according to the literature [22-31]. The in-hospital incidence combined with the advanced age of those infected, the high rate of medication and the high rate of comorbidities suggest that the infection typically occurs in frail patients.

E. faecalis was the most typical species isolated in this work. Furthermore, the prevalence of E. faecium was almost 50\%, appearing higher among patients in our study than reported in other studies [8-11]. An estimated 3.1\% of strains of E. faecalis and $22.2 \%$ of strains of E. faecium were resistant to ampicillin; moreover, resistance to gentamicin was found in $16.2 \%$ and $47.3 \%$ of strains, respectively.

The susceptibility reported for ampicillin is far from that reported in Asian literature [10], which reported susceptibility against $100 \%$ of E. faecalis isolates and from $3.8 \%$ up to $10.8 \%$ of E. faecium isolates. On the contrary, studies from Denmark and Canada reported susceptibility to ampicillin in $12.3 \%$ and $30 \%$ of E. faecium, respectively, and in $1.3 \%$ and $0.4 \%$ of $E$. faecalis, respectively [8,9].

Nonetheless, ampicillin-resistant E. faecium was not reported frequently in previous studies and outside Europe [2, 4, 8-11]. This data did not likely represent Italy's basic epidemiology $[3,5,7,11]$; therefore, more recent homogeneous studies are warranted.

Our study reported VRE in $2.2 \%$ of E. faecalis strains and $23.3 \%$ of E. faecium strains. The National Healthcare Safety Network in the US reported a higher rate, with $6.9 \%$ and $80 \%$ of vancomycin-resistant faecalis and faecium, respectively [2]. Among Danish and Canadian studies [8, 9], VRE data in E. faecalis were $1.5 \%$ and $1 \%$, respectively. These studies also reported lower rates of $E$. faecium VRE than us, 
Table 4 Multivariate analysis of risk factors for 21-day mortality-associated with Enterococcus spp. bacteraemia

\begin{tabular}{|c|c|c|c|c|c|c|c|c|c|}
\hline & \multicolumn{3}{|c|}{ Model 1} & \multicolumn{3}{|c|}{ Model 2} & \multicolumn{3}{|c|}{ Model 3} \\
\hline & HR & CI & $p$ & HR & CI & $p$ & HR & CI & $p$ \\
\hline Appropriate therapy & 1.44 & {$[0.79,2.63]$} & 0.23 & 1.39 & {$[0.76,2.53]$} & 0.289 & 1.16 & {$[0.64,2.08]$} & 0.628 \\
\hline Sex & 1.11 & {$[0.61,2.04]$} & 0.727 & 1.12 & {$[0.61,2.06]$} & 0.715 & 1.09 & {$[0.60,1.99]$} & 0.773 \\
\hline Age & 0.99 & {$[0.96,1.01]$} & 0.319 & 0.98 & {$[0.96,1.01]$} & 0.219 & 0.99 & {$[0.97,1.02]$} & 0.576 \\
\hline Charlson Index & 1.12 & {$[0.98,1.27]$} & 0.087 & 1.14 & {$[1.00,1.30]$} & 0.045 & 1.13 & {$[1.00,1.28]$} & 0.056 \\
\hline Solid organ transplant & 0.6 & {$[0.23,1.57]$} & 0.298 & 0.65 & {$[0.24,1.74]$} & 0.395 & 0.95 & {$[0.34,2.60]$} & 0.913 \\
\hline Use of steroids & 1.28 & {$[0.57,2.84]$} & 0.548 & 1.44 & {$[0.67,3.09]$} & 0.35 & 1.54 & {$[0.71,3.30]$} & 0.272 \\
\hline \multicolumn{10}{|l|}{ Polymicrobial } \\
\hline E. faecium vs. E. faecalis & 0.94 & {$[0.52,1.70]$} & 0.843 & 1.03 & {$[0.58,1.83]$} & 0.922 & 0.84 & {$[0.46,1.55]$} & 0.582 \\
\hline Shock (sepsis 3) & 3.82 & {$[1.53,9.55]$} & 0.004 & & & & & & \\
\hline Sepsis (sepsis 3) & 2.57 & {$[1.37,4.82]$} & 0.003 & & & & & & \\
\hline Shock (sepsis 2) & & & & 2.41 & {$[1.11,5.25]$} & 0.027 & & & \\
\hline Sepsis (sepsis 2) & & & & 0.50 & {$[0.26,0.99]$} & 0.047 & & & \\
\hline \multicolumn{10}{|l|}{ Severe sepsis } \\
\hline Vancomycin-resistant strains & & & & & & & 2.09 & {$[1.10,3.99]$} & 0.025 \\
\hline$N$ patients & 201 & & & 199 & & & 201 & & \\
\hline$N$ deaths & 53 & & & 53 & & & 53 & & \\
\hline
\end{tabular}

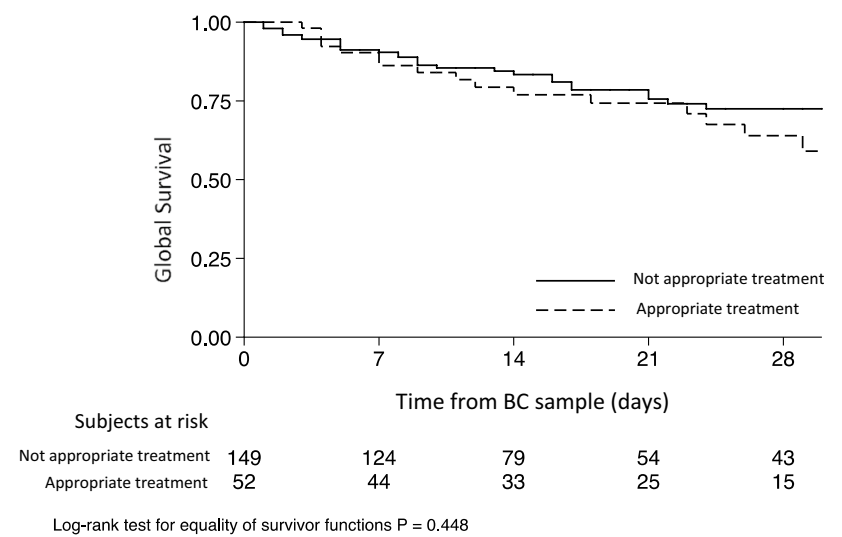

Fig. 1 Survival at 28 days from time from blood culture sample

at $1.3 \%$ and $7 \%$ in each group $[8,9]$. Antibiotic resistance for ampicillin and vancomycin in our study was higher for both E. faecalis and E. faecium than that reported in northern European and Canadian literature; however, it was far from the high rates in the US, notably for VRE strains $[2,4,8,9]$.

Although 59\% of patients in our study did not undergo echocardiography, the prevalence of endocarditis is in line with the literature where Enterococcus spp. is well known to be one of the most frequent causes of endocarditis [12]. Many infectious disease consultations discuss the importance of defining the source of infection, initiating appropriate antimicrobial therapy, managing treatment duration and de-escalation of the therapy once culture isolates are obtained [32, 33].
However, the management of BSI does not always occur after an infection specialist advises it $[32,33]$. We demonstrated in enterococcal endocarditis a significant difference in survival when appropriate treatment is administered. Unfortunately, we did not adjust for inappropriate therapy as a confounding factor for survival. Appropriate case management driven by infectious diseases consults includes repeated cultures to assess clearance and ultrasonography to rule out endocarditis in E-BSI [32,33]. These approaches might also have a role in mortality as previously shown [8-11].

The second aim of this study was to determine if combination therapy decreases mortality in patients with E-BSI. Only one out of four patients received appropriate therapy because clinicians stopped the therapy early; they decided only to use one drug or choose a drug not active against Enterococcus spp. To define the role of combination therapy, we identified differences between the number of patients who received appropriate therapy and those who received inappropriate therapy. Each variable was associated with choosing one of the two therapeutic regimens, except for diagnosed endocarditis which was more frequent in the group that received appropriate therapy. This finding can be explained considering that the length of treatment for endocarditis, antimicrobial choice and combinations could rely on many studies and guidelines [18-21].

At the univariate and multivariate analysis, we confirmed some risk factors for mortality previously described in the literature, such as resistance to vancomycin, sepsis and septic shock $[13,14]$. On the contrary, appropriate antibiotic therapy was not associated with lower mortality in our casuistic analysis. This finding is likely since the enrolled 
patients who received appropriate therapy could still have a poor outcome due to their multiple comorbidities. Therefore, the high medicalization and frailty of these patients may be confounding factors. Furthermore, we found that sepsis seems to be protective for mortality: this result could be due to the fact that Sepsis- 2 criteria excluded more critically ill patients. Unfortunately, we are not able to control the confounding factor of the use of combination therapy in patients with sepsis.

A survival of 28 days in $69.94 \%$ of patients seems to be higher than in other studies, in which a 21-day mortality was estimated in $21.4-24.8 \%$ of participants [13, 14]. However, it is necessary to consider that extensive literature has valued a 30-day survival rate showing a range of mortality from $21 \%$ up to $34 \%$ to be higher for $E$. faecium bacteraemia [13, 14].

There are several limitations to this study. First, this is a single-centre study that may not accurately reflect the general demographics of Italy. Second, the recurrence of BSI, surveillance blood culture and performance of surgical source control, especially in intraabdominal infections, were other missing data. Moreover, recently EUCAST has warned about the use of daptomycin in the treatment of Enterococcus spp. with daptomycin MIC of $4 \mathrm{mg} / \mathrm{L}$, despite that the warning was published after the study design and after our data collection.

Our retrospective study demonstrates that many E-BSI sources in clinical practices within IMWs are not always adequately investigated, and the adequate rule-out of intracardiac vegetations lacks in E-BSI. Despite these elements, inappropriate therapy did not decrease survival in this population. We reported a higher rate of E. faecium than other studies. While the rate of ampicillin resistance was lower than expected, the VRE rate was higher than that of Asian and northern European countries [4, 6, 10]. This resistance pattern is consistent with the local epidemiology and the nosocomial acquisition of the infection for the large majority of our cohort. It reflects the microbiological counterpart of the features of the elderly comorbid population with a lengthy history of medicalization and recent previous antibiotic exposure that often populates the internist wards.

Funding Not applicable.

Availability of data and material Available on affordable request.

Code availability Not applicable.

\section{Declarations}

Conflict of interest All authors have no conflicts of interest to declare.

Human and animal rights statement Data acquisition and analysis was performed in compliance with protocols approved by the Ethi- cal Committee of all the participant centers (Città della Salute e della Scienza, Turin, Piedmont, Ethics Committee; Ethical approval number 0031285). The study was conducted according to the guidelines of the Declaration of Helsinki.

Informed consent For this type of study, formal consent is not required.

\section{References}

1. Woodford N, Livermore DM (2009) Infections caused by Grampositive bacteria: a review of the global challenge. J Infect 59(Suppl 1):S4-16. https://doi.org/10.1016/S0163-4453(09) 60003-7

2. 2015-2017 Adult Antimicrobial Resistance (AR) Report Online Supplement. https://www.cdc.gov/nhsn/pdfs/datastat/2015-17adult-ar-data-2-508.pdf. Accessed 08 Feb 2021

3. Monticelli J, Di Bella S, Giacobbe DR, Amato G, Antonello RM, Barone E et al (2021) Trends in the incidence and antibiotic resistance of enterococcal bloodstream isolates: a 7-year retrospective multicenter epidemiological study in Italy. Microb Drug Resist 27(4):529-535. https://doi.org/10.1089/mdr.2020.0147 (Epub 2020 Sep 18)

4. Mendes RE, Sader HS, Castanheira M, Flamm RK (2018) Distribution of main Gram-positive pathogens causing bloodstream infections in United States and European hospitals during the SENTRY Antimicrobial Surveillance Program (2010-2016): concomitant analysis of oritavancin in vitro activity. J Chemother 30(5):280-289. https://doi.org/10.1080/1120009X.2018.1516272

5. Giacobbe DR, Battaglini D, Ball L, Brunetti I, Bruzzone B, Codda G, Crea F, De Maria A, Dentone C, Di Biagio A, Icardi G, Magnasco L, Marchese A, Mikulska M, Orsi A, Patroniti N, Robba C, Signori A, Taramasso L, Vena A, Pelosi P, Bassetti M (2020) Bloodstream infections in critically ill patients with COVID-19. Eur J Clin Investig 50(10):e13319. https://doi.org/ 10.1111/eci.13319 (Epub 2020 Aug 11)

6. Xie O, Slavin MA, Teh BW, Bajel A, Douglas AP, Worth LJ (2020) Epidemiology, treatment and outcomes of bloodstream infection due to vancomycin-resistant enterococci in cancer patients in a vanB endemic setting. BMC Infect Dis 20(1):228. https://doi.org/10.1186/s12879-020-04952-5.PMID:32188401; PMCID:PMC7079500

7. Luzzaro F, Ortisi G, Larosa M, Drago M, Brigante G, Gesu G (2011) Prevalence and epidemiology of microbial pathogens causing bloodstream infections: results of the OASIS multicenter study. Diagn Microbiol Infect Dis 69(4):363-369

8. Billington E, Phang S, Gregson D, Pitout J, Ross T, Church D et al (2014) Incidence, risk factors, and outcomes for Enterococcus spp. blood stream infections: a population-based study. Int J Infect Dis 26:76-82

9. Pinholt M, Østergaard C, Arpi M, Bruun N, Schønheyder H, Gradel K et al (2014) Incidence, clinical characteristics and 30-day mortality of enterococcal bacteraemia in Denmark 20062009: a population-based cohort study. Clin Microbiol Infect 20(2):145-151

10. Suzuki H, Hase R, Otsuka Y, Hosokawa N (2017) A 10-year profile of enterococcal bloodstream infections at a tertiary-care hospital in Japan. J Infect Chemother 23(6):390-393

11. Giovannenze F, Murri R, Palazzolo C, Taccari F, Camici M, Spanu T, Posteraro B, Sanguinetti M, Cauda R, Onder G, Fantoni M (2021) Predictors of mortality among adult, old and the oldest old patients with bloodstream infections: an age comparison. Eur J Intern Med. https://doi.org/10.1016/j.ejim.2020.12.017 (Epub ahead of print) 
12. Pericàs JM, Llopis J, Muñoz P, Gálvez-Acebal J, Kestler M, Valerio M, Hernández-Meneses M, Goenaga MÁ, Cobo-Belaustegui M, Montejo M, Ojeda-Burgos G, Sousa-Regueiro MD, de Alarcón A, Ramos-Martínez A, Miró JM, GAMES Investigators (2020) A contemporary picture of enterococcal endocarditis. J Am Coll Cardiol 75(5):482-494. https://doi.org/10.1016/j.jacc.2019.11.047 (Erratum in: J Am Coll Cardiol. 2020 Jun 16;75(23):2998-3000)

13. DiazGranados C, Zimmer S, Mitchel K, Jernigan J (2005) Comparison of mortality associated with vancomycin-resistant and vancomycin-susceptible enterococcal bloodstream infections: a meta-analysis. Clin Infect Dis 41(3):327-333

14. Prematunge $\mathrm{C}$, MacDougall C, Johnstone J, Adomako K, Lam F, Robertson J et al (2015) VRE and VSE bacteremia outcomes in the era of effective VRE therapy: a systematic review and metaanalysis. Infect Control Hosp Epidemiol 37(01):26-35

15. Singer M, Deutschman CS, Seymour CW et al (2016) The third international consensus definitions for sepsis and septic shock (sepsis-3). JAMA 315(8):801-810. https://doi.org/10.1001/jama. 2016.0287

16. Charlson M, Szatrowski TP, Peterson J, Gold J (1994) Validation of a combined comorbidity index. J Clin Epidemiol 47(11):12451251. https://doi.org/10.1016/0895-4356(94)90129-5

17. Dellinger RP, Levy MM, Rhodes A et al, Surviving Sepsis Campaign Guidelines Committee Including the Pediatric Subgroup (2013) Surviving Sepsis Campaign: international guidelines for management of severe sepsis and septic shock: 2012. Crit Care Med 41(2):580-637

18. Graninger W, Ragette R (1992) Nosocomial bacteremia due to Enterococcus faecalis without endocarditis. Clin Infect Dis 15(1):49-57. https://doi.org/10.1093/clinids/15.1.49

19. Shlaes DM, Levy J, Wolinsky E (1981) Enterococcal bacteremia without endocarditis. Arch Intern Med 141(5):578-581

20. Maki DG, Agger WA (1988) Enterococcal bacteremia: clinical features, the risk of endocarditis, and management. Medicine (Baltim) 67(4):248-269

21. Baddour LM, Wilson WR, Bayer AS, Fowler VG Jr, Tleyjeh IM, Rybak MJ et al (2015) American Heart Association Committee on Rheumatic Fever, Endocarditis, and Kawasaki Disease of the Council on Cardiovascular Disease in the Young, Council on Clinical Cardiology, Council on Cardiovascular Surgery and Anesthesia, and Stroke Council. Infective endocarditis in adults: diagnosis, antimicrobial therapy, and management of complications: a scientific statement for healthcare professionals from the American Heart Association. Circulation 132(15):1435-1486. https://doi.org/10.1161/CIR.0000000000000296 (Epub 2015 Sep 15) (Erratum in: Circulation. 2015 Oct 27;132(17):e215. Erratum in: Circulation. 2016 Aug 23;134(8):e113. Erratum in: Circulation. 2018 Jul 31;138(5):e78-e79)

22. Bright M, Parfitt EC, Pasquill K, Steele L, Laupland KB (2020) Occurrence and determinants of enterococcal bloodstream infections: a population-based study. Infect Dis (Lond) 52(9):638-643. https://doi.org/10.1080/23744235.2020.1774074 (Epub 2020 Jun 9)

23. Inghammar M, Engström G, Ljungberg B, Löfdahl CG, Roth A, Egesten A (2014) Increased incidence of invasive bacterial disease in chronic obstructive pulmonary disease compared to the general population-a population based cohort study. BMC Infect Dis 25(14):163. https://doi.org/10.1186/1471-2334-14-163

24. Wang Y, Oppong TB, Liang X, Duan G, Yang H (2020) Methicillin-resistant $S$. aureus and vancomycin-resistant Enterococci co-colonization in patients: a meta-analysis. Am J Infect Control 48(8):925-932. https://doi.org/10.1016/j.ajic.2019.11.010 (Epub 2019 Dec 19)

25. Papadimitriou-Olivgeris M, Drougka E, Fligou F, Kolonitsiou F, Liakopoulos A, Dodou V, Anastassiou ED, Petinaki E, Marangos M, Filos KS, Spiliopoulou I (2014) Risk factors for enterococcal infection and colonization by vancomycin-resistant enterococci in critically ill patients. Infection 42(6):1013-1022. https://doi.org/ 10.1007/s15010-014-0678-1 (Epub 2014 Aug 21)

26. Weber S, Hogardt M, Reinheimer C, Wichelhaus TA, Kempf VAJ, Kessel J, Wolf S, Serve H, Steffen B, Scheich S (2019) Bloodstream infections with vancomycin-resistant enterococci are associated with a decreased survival in patients with hematological diseases. Ann Hematol 98(3):763-773. https://doi.org/10.1007/ s00277-019-03607-z (Epub 2019 Jan 21)

27. Nielsen XC, Chen M, Hellesøe AM, Jeppesen PB, Gyldenlykke J, Tvede M, Andersen LP (2012) Etiology and epidemiology of catheter related bloodstream infections in patients receiving home parenteral nutrition in a gastromedical center at a tertiary hospital in Denmark. Open Microbiol J 6:98-101. https://doi.org/10.2174/ 1874285801206010098 (Epub 2012 Nov 30)

28. Cotogni P, Pittiruti M, Barbero C, Monge T, Palmo A, Boggio Bertinet D (2013) Catheter-related complications in cancer patients on home parenteral nutrition: a prospective study of over 51,000 catheter days. JPEN J Parenter Enteral Nutr 37(3):375383. https://doi.org/10.1177/0148607112460552 (Epub 2012 Sep 20)

29. Opilla M (2008) Epidemiology of bloodstream infection associated with parenteral nutrition. Am J Infect Control 36(10):S173. e5-S173.e8. https://doi.org/10.1016/j.ajic.2008.10.007

30. Giannella M, Bartoletti M, Gatti M, Viale P (2020) Advances in the therapy of bacterial bloodstream infections. Clin Microbiol Infect 26(2):158-167. https://doi.org/10.1016/j.cmi.2019.11.001 (Epub 2019 Nov 14)

31. Patterson JE, Sweeney AH, Simms M et al (1995) An analysis of 110 serious enterococcal infections. Epidemiology, antibiotic susceptibility, and outcome. Medicine 74(4):191-200. https://doi. org/10.1097/00005792-199507000-00003

32. Lee RA, Vo DT, Zurko JC, Griffin RL, Rodriguez JM, Camins $\mathrm{BC}$ (2020) Infectious diseases consultation is associated with decreased mortality in enterococcal bloodstream infections In: OFID. https://doi.org/10.1093/ofid/ofaa06

33. Rosselli Del Turco E, Bartoletti M, Dahl A, Cervera C, Pericàs JM (2021) How do I manage a patient with enterococcal bacteraemia? Clin Microbiol Infect 27(3):364-371. https://doi.org/10.1016/j. cmi.2020.10.029 (Epub 2020 Nov 2)

Publisher's Note Springer Nature remains neutral with regard to jurisdictional claims in published maps and institutional affiliations. 